\title{
Fabrication of magnetic ring structures for Lorentz electron microscopy
}

\author{
L.J. Heyderman ${ }^{\mathrm{a}, *}$, M. Kläui ${ }^{\mathrm{b}}$, R. Schäublinc, U. Rüdiger ${ }^{\mathrm{b}}$, C.A.F. Vaz ${ }^{\mathrm{d}}$, \\ J.A.C. Bland ${ }^{\mathrm{d}}$, C. David ${ }^{\mathrm{a}}$ \\ ${ }^{a}$ Laboratory for Micro- and Nanotechnology, Paul Scherrer Institut, Villigen PSI, CH-5232, Switzerland \\ ${ }^{\mathrm{b}}$ Universität Konstanz, Universitätsstr. 10, Konstanz, Germany \\ ${ }^{\mathrm{c}}$ Fusion Technology-Materials, CRPP-EPFL, Villigen PSI, CH-5232, Switzerland \\ ${ }^{\mathrm{d} C a v e n d i s h ~ L a b o r a t o r y, ~ U n i v e r s i t y ~ o f ~ C a m b r i d g e, ~ M a d i n g l e y ~ R o a d, ~ C a m b r i d g e, ~ C B 3 ~ O H E, ~ U K ~}$
}

\begin{abstract}
We have fabricated three-quarter ring structures on silicon nitride membranes for magnetic domain imaging using Lorentz electron microscopy. The ring structures have linewidths of 100, 250, 350 and $700 \mathrm{~nm}$, and outer diameters of $1.65 \mu \mathrm{m}$. Using the Foucault imaging mode, we have observed the magnetic domain configurations in ring structures with an inter-ring separation of $100 \mathrm{~nm}$. Head-to-head transverse domain walls and vortices are seen depending on the ring geometry and stray field interactions between the rings. It is also possible to see contrast due to the stray field produced by transverse walls.
\end{abstract}

Keywords: Ferromagnetic rings; Lorentz microscopy; Head-to-head domain wall; Domain wall interactions

The current interest in nanoscale magnetic elements is driven by both the interesting physics at reduced lateral dimensions and the possible industrial applications. For such applications, it is important to have precise control of the magnetic switching, which can be achieved when the magnetic elements have well-defined and reproducible remanent states and the switching processes are simple. The ring geometry has so far displayed these desired characteristics and has a good potential for use in high-density data storage [1,2] and bio-detection [3].

The rings have particularly simple magnetic states due to their high symmetry. In particular, in narrow rings

\footnotetext{
*Corresponding author. Tel.: + 41623102613 ; fax: +41623102646.

E-mail address: laura.heyderman@psi.ch (L.J. Heyderman).
}

there are two stable magnetic states [4-6]; the fluxclosure vortex state, and the 'onion' state, accessible reversibly from saturation and characterised by the presence of two opposite head-to-head walls. These can either be transverse or vortex walls, schematically shown in Figs. 1a and $\mathrm{b}$ respectively, depending on the ring geometry [7]. Thus two transitions occur in the rings; at intermediate fields the rings switch from the onion to the vortex state and at high fields they switch from the vortex to the opposite onion state $[4,8]$. In order to fully understand the detailed magnetic behaviour of such rings, it is important to carry out direct observations of the magnetic domain structures. From such observations the dependence of the domain structures on the ring geometry can be determined, making it possible to tailor their magnetic properties. In addition to scanning 


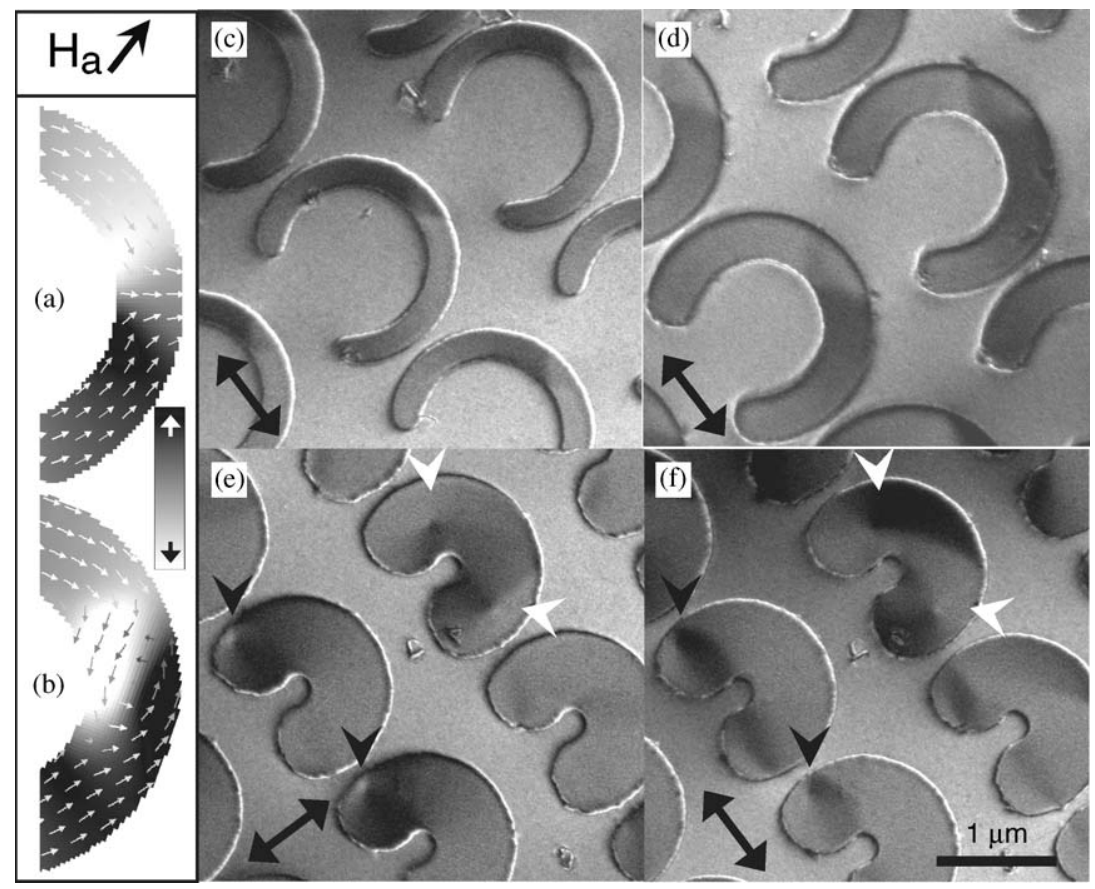

Fig. 1. (a,b) Schematic representations of magnetic spin structures and resulting magnetic contrast for a transverse and a vortex domain wall, respectively. (c-f) Foucault images of three-quarter rings after saturating the sample with an applied field, $H_{\mathrm{a}}$, whose orientation is indicated: (c) $W=250 \mathrm{~nm}$, (d) $W=350 \mathrm{~nm}$ and (e, f) pair of images for $W=700 \mathrm{~nm}$ with the positions of vortices indicated by the four single arrowheads. The images are sensitive to the direction of the magnetic induction component given by the double-headed arrows.

electron microscopy with polarisation analysis (SEMPA) and photoemission electron microscopy (PEEM) analysis [9], transmission electron microscopy (TEM) can provide detailed information on the magnetic spin structures. We have therefore fabricated ferromagnetic ring structures on substrates for Lorentz electron microscopy [10]. Here, a magnetic field can be applied to the sample using the objective lens field, which can be varied by changing the lens current or tilting the sample.

In order to produce samples suitable for TEM observations, the ferromagnetic rings must be fabricated on membranes to allow the transmission of electrons. While we have already shown that it is possible to prepare magnetic rings down to outer diameters of $90 \mathrm{~nm}$ using electron lithography with a lift-off process [11], the lift-off requires removal of the unwanted resist and magnetic material in an ultrasound bath. This is not compatible with membrane substrates because the membranes are too fragile to survive the ultrasound treatment. We report here on the fabrication of lift-off ring structures on membranes without the use of ultrasound and the observation of the magnetic domain structures using the Foucault imaging mode.

We employed electron beam lithography to fabricate magnetic rings on silicon nitride membrane substrates (from Silson Ltd, UK) suitable for TEM. The substrates have $500 \mu \mathrm{m}$ square membranes with a thickness of $50 \mathrm{~nm}$, back-coated with $5 \mathrm{~nm} \mathrm{Cr}$ to minimise charging during the electron beam writing. The front side of the substrate was spin-coated with $100 \mathrm{~nm}$ of poly methylmethacrylate (PMMA) resist and the resist was patterned with ring structures using a Leica LION LV1 electron beam writer with the electron beam energy set to $2.5 \mathrm{keV}$ to minimise the proximity effect. To write the ring structures, the electron beam follows a circular single pixel path along the ring and the linewidth is determined by the electron beam dose and defocus [12]. We created $6 \times 6$ arrays of full rings and three-quarter rings where a quarter of the ring was missing resulting in a horseshoe shape. These ring structures were produced with linewidths of $W=100,250,350$ and $700 \mathrm{~nm}$, and an outer diameter of $1.65 \mu \mathrm{m}$. The inter-ring spacing could be changed to vary the magnetic stray field interaction between the rings. $10 \mathrm{~nm}$-thick polycrystalline permalloy $(\mathrm{NiFe})$ films, with a $2 \mathrm{~nm}$ thick gold capping layer to prevent oxidation of the magnetic film, were deposited on the patterned resist by MBE evaporation in an ultra-high vacuum deposition chamber at a base pressure of $3 \times 10^{-10}$ mbar [6]. The lift-off process involved removal of unwanted resist and magnetic material in acetone. For the full rings, the isolated centre of the ring did not lift off without the use 
of ultrasound. For the three-quarter rings, the material lifted off as one piece. While the switching properties of the three-quarter rings are different from that of the full rings, it was possible to use the three-quarter rings to investigate the magnetic domain walls and domain wall interactions.

We employed the Foucault imaging mode to observe the magnetic domain structures in the three-quarter rings. In the current work, we concentrate on ring arrays with an inter-ring separation of $100 \mathrm{~nm}$ which provides a ring separation small enough to observe the effect of magnetic stray field interactions between adjacent rings. Imaging was carried out using a JEOL $2010 \mathrm{LaB}_{6}$ TEM with a high-tilt lens operated at $200 \mathrm{kV}$ and the objective lens field could be turned off by switching to the low magnification mode. The objective lens field, which is almost vertical, is used to saturate the sample along a given orientation with respect to the ring structures by tilting the sample by $30^{\circ}$ about an axis perpendicular to the required orientation. The remanent states were then observed after removal of the magnetic field.

We first applied a field to produce a single head-tohead domain wall. The orientation of the applied field, $H_{\mathrm{a}}$, with respect to the ring structures is given in Fig. 1 and the expected contrast for typical transverse and vortex walls is indicated in the schematic diagrams in
Figs. 1a and b, respectively. Here it can be seen that the transverse wall is associated with a triangular domain and that the magnetic contrast associated with the vortex wall reflects the circulating magnetic spins. For the rings with $W=250$ and $350 \mathrm{~nm}$, transverse walls were clearly observed (see Figs. 1c and d). For $W=$ $100 \mathrm{~nm}$, the detailed wall structure was difficult to ascertain due to the low magnetic contrast. However, since transverse walls become more and more favourable with decreasing linewidth [13] and from the weak contrast available, we can conclude that they are also transverse walls. For the rings with large linewidths, $W=700 \mathrm{~nm}$, several different remanent domain states can be supported, some of which can be seen Figs. 1e and $f$, which is a pair of Foucault images sensitive to orthogonal magnetisation induction components. The upper ring structure in Figs. 1e and $\mathrm{f}$ supports two vortices (indicated by the two single white arrowheads) and the other rings show vortices pinned at the ring edge (indicated by the two single black arrowheads).

We then applied a field at an orthogonal orientation to induce two head-to-head walls (see Fig. 2) which is equivalent to the onion state in full rings. For $W=250$ and $100 \mathrm{~nm}$, all walls are transverse walls (see Figs. 2a and c). For $W=350 \mathrm{~nm}$, the effect of stray field interactions between adjacent rings becomes apparent

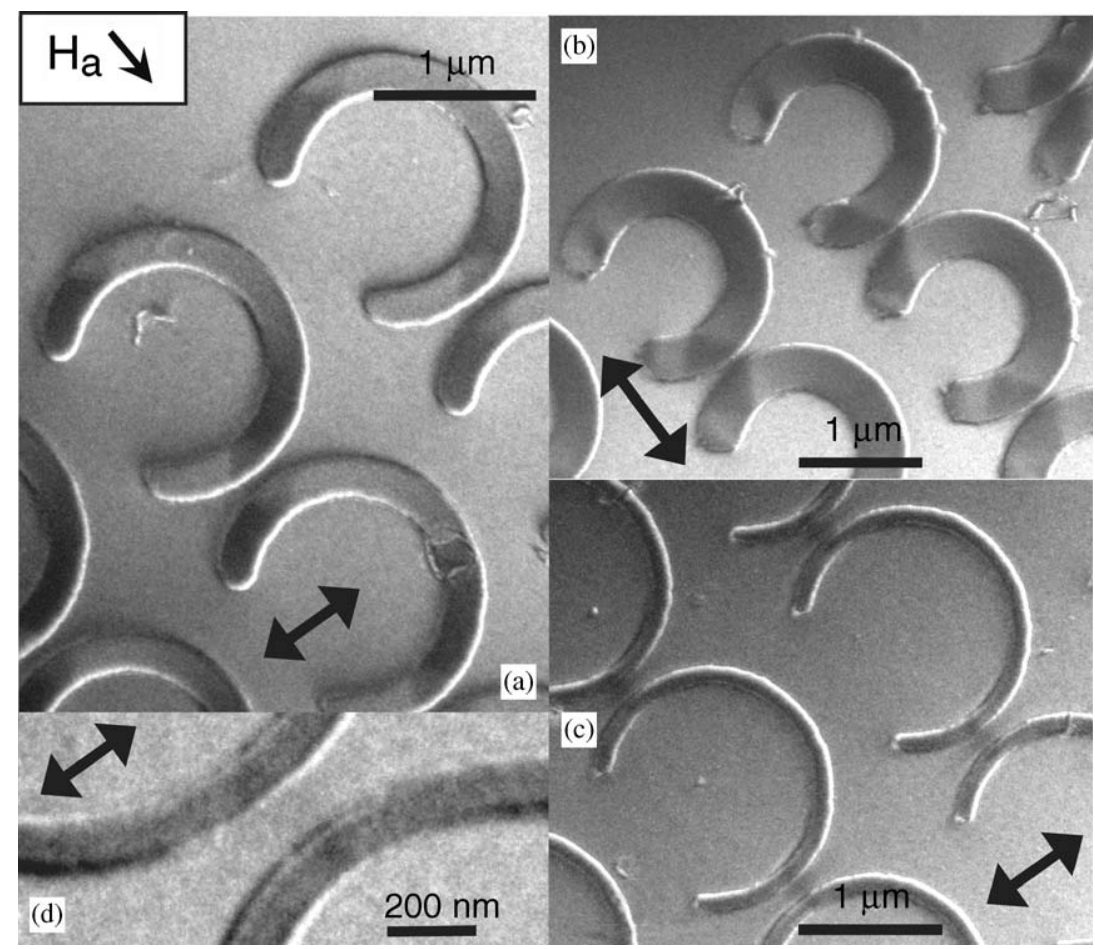

Fig. 2. Foucault images of three-quarter rings after saturating the sample with an applied field, $H_{\mathrm{a}}$, whose orientation is indicated: (a) $W=250 \mathrm{~nm}$; (b) $W=350 \mathrm{~nm}$; and (c,d) $W=100 \mathrm{~nm}$. The images are sensitive to the direction of the magnetic induction component given by the double-headed arrows. 
(Fig. 2b). At the edge of the array a vortex forms at the line-end, rather than a transverse wall as seen in Fig. 1d, as a result of the local geometry. However close to all other neighbouring line-ends, transverse walls are present and are favoured as a result of the stray fields associated with the transverse walls. The contrast resulting from the stray fields between two adjacent transverse walls can also be detected, as clearly seen for $W=100 \mathrm{~nm}$ in Fig. 2c. A close-up of these walls shows that the contrast of the transverse walls in these narrow lines is just discernible (Fig. 2d).

We have seen that for three-quarter rings with a linewidth of $W=350 \mathrm{~nm}$, transverse walls are favoured over vortices in the vicinity of line-ends in neighbouring rings as a result of the stray field interactions. Such interactions will determine the phase diagram of walltype versus ring geometry and spacing [14]. This is not only of scientific interest but is vital for magnetic storage devices where the demand for high densities requires an understanding of the effects of stray field interactions between neighbouring magnetic elements with small separations.

The authors would like to thank the staff at the Laboratory for Micro- and Nanotechnology, Paul Scherrer Institut, for their support with fabrication, and the EU project MASSDOTS (\#22464), the Gottlieb Daimler and Carl Benz Foundation, DFG SFB 513 and DAAD for financial support.

\section{References}

[1] G.A. Prinz, J. Magn. Magn. Mater. 200 (1999) 57.

[2] J.-G. Zhu, Y. Zheng, G.A. Prinz, J. Appl. Phys. 87 (2000) 6668.

[3] M.M. Miller, G.A. Prinz, S.-F. Cheng, S. Bounnak, Appl. Phys. Lett. 81 (2002) 2211.

[4] J. Rothman, M. Kläui, L. Lopez-Diaz, C.A.F. Vaz, A. Bleloch, J.A.C. Bland, Z. Cui, R. Speaks, Phys. Rev. Lett. 86 (2001) 1098.

[5] M. Kläui, J. Rothman, L. Lopez-Diaz, C.A.F. Vaz, J.A.C. Bland, Z. Cui, Appl. Phys. Lett. 78 (2001) 3268.

[6] M. Kläui, C.A.F. Vaz, J.A.C. Bland, W. Wernsdorfer, G. Faini, E. Cambril, Appl. Phys. Lett. 81 (2002) 108.

[7] M. Kläui, C.A.F. Vaz, L. Lopez-Diaz, J.A.C. Bland, J. Phys.: Condens. Matter 15 (2003) R985.

[8] M. Kläui, L. Lopez-Diaz, J. Rothman, C.A.F. Vaz, J.A.C. Bland, Z. Cui, J. Magn. Magn. Mater. 240 (2002) 7.

[9] M. Kläui, C.A.F. Vaz, J.A.C. Bland, T.L. Monchesky, J. Unguris, E. Bauer, S. Cherifi, S. Heun, A. Locatelli, L.J. Heyderman, Z. Cui, Phys. Rev. B 68 (2003) 134426.

[10] J.N. Chapman, J. Phys. D 17 (1984) 623.

[11] L.J. Heyderman, C. David, M. Kläui, C.A.F. Vaz, J.A.C. Bland, J. Appl. Phys. 93 (2003) 10011.

[12] C. David, D. Hambach, Microelectron. Eng. 219 (1999) 46.

[13] R.D. McMichael, M.J. Donahue, IEEE Trans. Magn. 33 (1997) 4167.

[14] M. Klaui, et al., unpublished. 